\title{
Management Measures of Public Bicycle System
}

\author{
MA yue ${ }^{1, a}$ ZHU Xiao-ning ${ }^{2, b}$ \\ ${ }^{1}$ Room 1102, Building 8, JiaoDadong Road 18, HaiDian Distict, BeiJing 100044 China \\ ${ }^{2}$ Room 603, School of Traffic and Transportation, Beijing Jiaotong University, BeiJing 100044 \\ China \\ a11120977@bjtu.edu.cn, ${ }^{\text {bxnzhu@njtu.edu.cn }}$
}

Keywords: Planning; Public bicycle; Management measures

\begin{abstract}
Good urban planning can make people have a healthy life style. Otherwise the establishment of urban structure once, wanting to change the impact of urban layout to urban traffic is difficult. When planning, consider walking, bicycle, public transport, car and so on by turn. Give the special opinions for the implementation prospects, to say that the bicycle will have a high demand in the future. From the points of propaganda risk, policy risk and financial risk say the risk of public bicycle facing in the future. In order to decrease the degree of risk and have a good future, the system should strengthen the planning and management, social consciousness and propaganda, and management and operation. These measures will help the public bicycle system more reasonable, and make sure the system develops smoothly.
\end{abstract}

\section{Introduction}

The urban planning has a long and structural role on its development. The urban planning will affect the urban economy, because for the material environment, once established, it is difficult to change. Good urban planning can make people have a healthy life style, which can reduce the power consumption in the production, living and other aspects in order to reduce the emissions of carbon dioxide. Nowadays in some cities the traffic conditions are not very good, even can say very crowded. The mostly reason is that when planning transportation is not considered well. The urban structure once established, it is difficult to change the traffic problems brought by the urban structure layout with some measures.

With the urban process accelerating the population constantly transfers to the urban leading to the urban expanding to the surrounding area. It expands the range of life. In the surrounding of urban owing to the public traffic is poor, the travel distance of people is always long. Some people are unwilling to choose the public traffic with poor service, which makes the ratio of car higher. The residential area planning and design have a clear and obvious boundary, and the management of entrance is basically closed. The public traffic not benefiting for the people travel is excluded outside the residential area. The residential area is too big, to some extent, which encourages the car travel and limits the walking and bicycle travel. Meanwhile in our country the planning owners belong to different administration organizations resulting in the bad cohesions in longitudinal and horizontal planning, which make a serious influence on the integrity of planning.

Researches generally show that the common laws of sustainable traffic land used planning are that reducing the demand of travel and the distance of travel, supporting walking, bicycle, public traffic, and then limiting the use of car. Our country puts forward the policy of the priority to the development of public traffic, under the premise of the priority of important traffic corridor. The comprehensive public traffic all priority is difficult to achieve. According to the situation of today's urban and the development policy of future urban, the construction of urban should firstly be helpful for walking and bicycle using, encourage the public traffic with high performance-price ratio, at the same time pay more attention on improving the urban image and controlling the development of car. In other words urban construction firstly considers walking construction with good environment, secondly considers the bicycle construction to use conveniently, in these bases advocate the construction of public traffic, and finally considers the urban image improving engineer and development of car travel. 
In the whole transportation especially focus on the bicycle travel, nowadays even if the bicycle is common in the urban, the transfer ratio of bicycle still lower. In our country many cities have or begin to build rail transit system. The cost of the construction is very high. If the bicycle system can combine to the rail transit system, it not only reduces the cost of system construction, but also expands the service radius of system.

Relative to the public traffic with fixed-point, alignment and timing, bicycle travel has big flexibility. Bicycle can enter small lanes while public travel not. According to statistics, sometimes for traffic jams and other factors, the motor travel always needs more time than bicycle travel. In our country the layout of many cities are single center. The radius of urban land is $6-10 \mathrm{~km}$. In this range bicycle travel always has many advantages. On basis of our country economic level and living condition, single center will last a long period. With the expansion of urban area, the demand of bicycle travel will transfer to other transportations, but meanwhile new short distance demand of bicycle travel will occur.

\section{Prospects}

A rising trend of daily short transport. For the public transport "last mile" problem, public bicycle has a good advantage, which can make up the insufficient of public transport not arriving at the destination and be the short transport substitute for walking. It will be favor for people. Because of some residential area being too big, and the problem of public traffic planning, some public traffic lane cannot arrive at the destination of people. This leads to the people having a long distance to walk, even giving up the choice of using public traffic, and choosing the car travel. The establishment of public bicycle solves this problem in some extent.

Demands increasing when to or off working. High peak is during the time people going to or off working. This lets the urban traffic having been crowded more load, and the traffic flow is slow. Working people of short distance experience a long time delayed on the road, then they more and more apt to the convenient flexible bicycle travel. Bicycle has a small size, without the lane limiting, and has a high flexibility. In one word the establishment of public bicycle benefits for the people. It has many potential demands.

Aid tourism business optimization and development. Riding bicycle to tour is not only convenient to sightseeing scenery of any places, but is a manner to exercise. It is the choice of fashionable young people. In some tourism and recreational area the establishment of public bicycle can meet some people demands. Meanwhile bicycle travel that is a green, meets the standard of protection to the environment and reducing pollution. Riding bicycle can let the people enjoy the scenery and custom of visiting places at any time without considering the parking position. You need not to stop your feet.

\section{Risk Prediction}

Propaganda risk. The change of consumers maybe result in the bicycle rent being shelved. Therefore this brings risks to the market of bicycle rent. However the changes of rivals will also make bicycle having competitive advantages stand out and occupy a large market share. The market is rapidly changeable. Not guarantee that the consumers demand and the competitors whether or not change, and when to change. So we must make a complete set of effective propaganda strategies to avoid the potential risks of market.

Policy risk. Although government at present advocates the green travel, encourage the public traffic and strongly support the bicycle rental industry. With the rapid development of economy, life rhythm speeding up and the improvement of environment gradually, the policy maybe have some changes. Nowadays people pay more attention on environment protection and sustainable development, but with the time passing it is hard to say the policy whether or not will tilt.

Financial risk. Financial risk is a real problem that the public bicycle system must face during the management process. This risk is objective existence, only through taking effective measures to reduce the risk, but not completely eliminate the risk. The establishment of public bicycle system is to alleviate 
the urban traffic congestion. The establishment and maintenance of the system need much money, if only depending on the rent to maintain the system is hard. It needs to undertake financial risks.

\section{The Management Measures of Public Bicycle}

The prospect of public bicycle system is more optimistic, but there are still all kinds of risks. Trying to reduce the risks, improve the feasibility of implementation and strengthen the management measures of the system. Specific measures are as follows:

1. Enhance planning and management. In order to enhance planning and management it will increase the degree of the following four aspects, namely the infrastructure settings related with bicycle, the road setting of motor and bicycle, lease point planning and the cooperation with related department.

1) Strengthen the infrastructure settings related with bicycle. The whole urban planning should based on the development situation and trend of urban traffic, the population and travel characteristics, and the policy of urban development and other comprehensive situations. On the basis of the specific conditions of different regions take different policies. In the urban center with high people flow intensity, maybe set up bicycle routes according to real conditions. Enhance the ability to gather or scatter the people flow in the urban center. Encourage the transfer and improve the utilization ratio of public traffic. However outside the downtown the environment of bicycle travel is bad. The land resource is relatively loose. In order to be sure the safety of bicycle users, maybe consider to set up the special-purpose roads for the bicycle. So that the interference between motors and bicycles is reduced, this enhances the security, and at the same time improves the travel efficiency. But in the suburb of the urban, because of the distance from working area usually is further. The travel means cannot be only the bicycle, can choose public traffic to transfer, so as to improve the travel efficiency.

2) The road setting of motor and bicycle. In our urban the road settings with the motor and bicycle separated are less. Even if the road is separated, when into the practice, only less people abide. It often happens that motors preempting the road of bicycles. What's more, the road management is not serious in our country. This contributes to the phenomenon accruing. The phenomenon of mixed traffic is serious on some roads. This always leads to the traffic accidents happening. With the number of motors increasing, the bicycle road becomes more and more narrow. Mixed traffic decreases the road cap urban, and also brings potential security dangers. Set up the bicycle route or specific-purpose road for bicycle on both sides of the motor road based on the actual situation. The road isolated can make the motors and bicycles travelling on their own roads. Meanwhile strengthen the road management. These measures let motors and bicycles isolated get thoroughly carry out.

3) Lease point planning. The position of lease points whether or not reasonable is the key factor to success. In order to be convenient for the users to quickly find lease points, the lease points should have clear signs and labels. The number of bicycles parking places in the lease points should be bigger than the number of bicycles. This will benefit the bicycles exchange storage during the lease points. The bicycles need not to be returned in the original point, increasing the bicycle liquidity and readjusting the number of bicycles with the direction and flow by the managers. It also leaves more space for further expansion.

4) Strengthen cooperation with related department. The public bicycle system involves many departments, such as planning, traffic even the manufacturers of bicycles. The cooperation and the degree of interest coordination between the departments depend on the system whether or not smoothly. Therefore, strengthen the coordination and communication during the departments. If there is a problem, timely reflect to the corresponding departments. How to divide the right of lease points land and how to bring the social capitals in order to decrease the risks of government and other problems all need to consult with the related departments. Strive to get the policy with many benefits.

2. Enhance the social consciousness and propaganda. In order to enhance the social consciousness and propaganda it will increase the degree of the following two aspects, namely the identification degree and the degree of propaganda. 
1) Increase identification degree. The urban develops more rapidly nowadays and the population is also more and more large. With the traffic more and more crowded, advocate the public traffic in order to ensure the travel efficiency. The bicycle travel as green traffic can make some related measures to play its advantages fully. Many people think that the bicycle is the performance of economic backwardness, and it can hinder our country developing forward. This wrong view leads the people reject the bicycle fundamentally. The utilization of bicycles is lower. Wanting to change this condition, firstly need to change the view to the bicycle. Prove the advantages of bicycles with the real and reliable data. Make people use the bicycle to travel consciously, and widen the market of public bicycle.

2) Strengthen the propaganda. The uses of bicycle are generally classified into the short trip, transfer and fitness. According to the purpose and characteristics of travel, propagandize with purpose. The public bicycle system cannot be implemented in some areas mainly because of the degree of propaganda is low. The people don't learn about the system, even know nothing. This results in the possibility of the system being killed. Strengthen the propaganda of the system through the following measures.

(1) Network propaganda. 21 century is the age of knowledge and economy, and the age of information. The advantage of network propaganda is more and more popular. The strategy of system propaganda should be put in the first place. Network spreads much faster. It is the most effective. Making use of the advantages of the network propaganda let many people know about the characteristics of bicycle system and the situation of specific implementation.

(2) Advertisement propaganda. We can see all kinds of advertisement from television, network even billboard on both sides of the road. We make full use of the characteristics of the advertisement covering most areas. This will attract more potential users. Whether the TV ads on the buses or the billboards beside the stations, these will attract the attention when people are in the bus or waiting for the bus. This can make the system get maximum propaganda.

(3) Reputation propaganda. To guarantee the service related with system, make the system form a good reputation. The system itself services the public people. Good reputation is necessary for the system lasting for a long time. The reputation once formed will contribute many people to use this characteristic. It will give many benefits to the system and cause the system marching smoothly.

(4) Price propaganda. The price of public bicycle system is low. We can utilize this advantage to make people use bicycle to travel. The travel means the change from the motor to the bicycle. Although in some areas the price of public traffic is lower such as in Beijing. But this just is some individual cities. The price of public traffic still is higher in most of cities. Rather than to say that the cars park at random because of no parking places, this causes the road crowded. The public bicycle attracts more consumers because of the cheap rental fee.

3. Reasonable management and operation. In order to make the system have the reasonable management and operation it will allocate bicycles scientifically and confirm the cost and deposit.

1) Allocate bicycles scientifically. The position of lease points whose function is various is different. For example the lease points locating in the residential area are convenient for life and work travel. But the bicycle flow and direction during a period time is certain directivity. This leads to the unbalance of bicycles. In order to ensure the system provide enough bicycles for the people, need the management to assist. Through the system monitor the condition of bicycles at any time, guarantee the reasonable allocation of the bicycles. However the lease points in the bus station or in the subway station are convenient for people to transfer. The people flow mostly and basically is balanced. So the number of bicycles can arrive at the dynamic balance.

2) Confirm the cost and deposit. How to confirm the cost and deposit of the system needs to consider seriously. The cost is too high to make the passenger flow turn to other transportations. The cost is so low that it can burden the system heavily. This will utilize one part of the capital to assist the system. How to confirm the degree of the cost and deposit affects the implementation of the system to a great extent. The degree should be in the range of being accepted. We can draw lessons from the thought of implementation of public bicycle in the foreign countries. 
Confirm the deposit and cost seriously. The confirmation of deposit and cost maybe make some adjustments according to the situation of system implementation and reaction of the residents. Guide the people use the bicycle in a reasonable time and distance. The cost once is set, the lower simply, the higher difficultly. The establishment of public bicycle system is the aim to service the people. The system can charge a small mount of cost. In essence the system is a welfare undertaking. Bicycle whose fees are divided into deposit and cost two parts is a short transportation.

(1) Deposit. The purpose of deposit one side is to ensure users return the bicycles on time and assure the bicycles; the second is to subsidize the fees of bicycle purchase and operation maintenance. So that it can decrease the press of finance. The deposit of the system should be the certain proportion of the bicycle cost. This not only makes the users give up the plan to purchase new bicycles, but the price should be higher than used bicycles in the black market preventing the bicycles being steal.

(2) Cost. At present many management companies take the policy on the basis of the difference of time. This policy encourages the efficiency of using bicycles. The cost is more or less depending on the price of the public traffic. So it arrives at the aim to scatter the passenger origin of public traffic.

3) Handle the rental card. The principles of public bicycle rental card are that: rent bicycles relying on the bus card or the citizen card opened, certain amount money should be put in the card, even can apply for the special rental card. For the permanent residents can handle the formalities in the lease points or the bus stations valid identity certificate.

\section{Summary}

The bicycle with convenience, speedy, cheap and other characteristics becomes the general public transportation. With the number of motors and the demand of people increasing rapidly, the characteristics of bicycles maybe have some new changes. The advantages of the bicycle system are low pollution and low consumption. The system during the implement depends on many aspects and many departments to cooperate. At the same time the system has many risks to analyze, and in this paper state the details of implementation so as to guarantee the system march smoothly.

In our country bicycle travel existing for a long time is necessary. Taking what are the measures for the bicycles such as how to plan the traffic facilities and take the management measures is important for the development of bicycle traffic. As the special transportation the public bicycle will be affected seriously. There are many aspects to study. The traffic characteristics of bicycles still have many things to research. With the constant changes the characteristics maybe have some changes. The related management measures need to be improved, so as to make the system more reasonable and more systematic.

\section{References}

[1] GTZ Germany Technology Cooperation Company: Sustainable Transport: A Sourcebook for Policy-makers in Developing Cities (People Traffic Press, 2005).

[2] Gong Dijia, Zhu Zhongdong: Implementation Mechanism of Urban Public Bicycle System. Urban Transport, VOL.6 (2008) NO.6.

[3] Wang Xiuxiu: Operation Mode of Public Bicycle in HangZhou.Transportation Enterprise Management, (2010) NO.5, p.2.

[4] Wu Wenzhi: Introduce Foreign Capital to Beijing Public Bicycle. Beijing Business News, (2008) NO.7. 